\title{
Statut juridique de l'enseignement universitaire de la théologie et droit local alsacien-mosellan
}

Jean-Marie Woehrling

\section{OpenEdition}

Édition électronique

URL : https://journals.openedition.org/rdr/1434

DOI : $10.4000 /$ rdr.1434

ISSN : 2534-7462

Éditeur

Presses universitaires de Strasbourg

\section{Édition imprimée}

Date de publication : 6 mai 2021

Pagination : 61-84

ISBN : 979-10-344-0089-8

ISSN : 2493-8637

\section{Référence électronique}

Jean-Marie Woehrling, «Statut juridique de l'enseignement universitaire de la théologie et droit local alsacien-mosellan », Revue du droit des religions [En ligne], 11 | 2021, mis en ligne le 06 mai 2021, consulté le 03 mai 2022. URL : http://journals.openedition.org/rdr/1434 ; DOI : https://doi.org/ $10.4000 /$ rdr. 1434

\section{(c) (1) (8)}

La revue du droit des religions est mise à disposition selon les termes de la Creative Commons Attribution - Pas d'Utilisation Commerciale 4.0 International - CC BY-NC 4.0. 


\section{Statut juridique de l'enseignement universitaire de la théologie et droit local alsacien-mosellan}

\section{Jean-Marie WOEHRLING}

Président de l'Institut du droit local alsacien-mosellan

\section{RÉSUMÉ}

En dehors de la non-application de la loi de 1905 et du maintien de la convention de 1902 entre l'État et le Saint-Siège, le droit alsacien-mosellan ne comporte pas de bases juridiques originales pour l'enseignement de la théologie à l'Université publique. C'est le contexte intellectuel et culturel local qui présente un cadre favorable à un tel enseignement en raison de l'existence des facultés de théologie catholique et protestante, ainsi que d'une tradition historique d'intérêt des autorités publiques pour la formation des cadres religieux. Les résistances ont empêché la création d'une faculté de théologie musulmane. Pourtant, aucun principe du droit constitutionnel ne fait obstacle au développement d'enseignements de type théologique à l'Université publique s'ils se conforment aux principes de l'enseignement universitaire.

\section{Abstract}

Apart from the non-application of the Law of 9 December 1905 and the maintenance of the 1902 Convention between the State and the Holy See, Alsatian-Moselle law does not include any original legal basis for the teaching of theology at public universities. It is the local intellectual and cultural context which provides a favourable framework for such teaching, owing to the existence of Catholic and Protestant theological faculties and a historical tradition of interest on the part of the public authorities in the training of religious leaders. Resistance has prevented the creation of a faculty of Muslim theology. However, no principle of constitutional law prevents the development of theology-type teaching at public universities if it conforms to the principles of university teaching. 
L 'existence de facultés de théologie catholique et protestante dans l'université de Strasbourg est perçue comme un aspect remarquable du droit local alsacien-mosellan. Il s'agit d'un des éléments de ce qu'on appelle le «statut local des cultes» dont les différentes composantes sont juridiquement autonomes, mais forment politiquement et sociologiquement un ensemble. Ce statut est marqué par une conception propre des relations entre l'État et les institutions cultuelles caractérisée par la non-introduction de la loi de Séparation du 9 décembre 1905 et par des engagements réciproques, dont l'un des aspects réside dans l'attention apportée par l'État à la formation de personnels religieux.

Dans la période récente, à savoir le dernier quart de siècle, le droit local des cultes, et notamment le droit de l'enseignement de la théologie à l'Université publique, a connu deux questionnements nouveaux:

- comment ce droit local peut-il prendre en compte des cultes autres que les cultes statutaires?

- quelles sont les contraintes résultant du cadre constitutionnel?

Ces questionnements ont conduit depuis quelque temps à procéder à un réexamen du droit de l'enseignement universitaire public de la théologie. C'est dans cette ligne que se situe la présente contribution. Elle se propose dans un premier temps de préciser le droit local applicable en la matière (1), puis d'analyser le cadre formé par le droit constitutionnel (2).

\section{LE DROIT LOCAL EN MATIÈRE D’ENSEIGNEMENT UNIVERSITAIRE DE LA THÉOLOGIE}

En quoi consiste le «droit local de l'enseignement universitaire de théologie» ? Le contenu juridique exact de ce régime particulier n'est pas clair. Il faut essayer de procéder à un inventaire. Parmi les règles juridiques susceptibles de concerner cet enseignement, on distinguera entre les dispositions de droit général non introduites (1.1) et les dispositions propres à l'Alsace et à la Moselle (1.2).

\subsection{DISPOSITIONS DE DROIT GÉNÉRAL NON INTRODUITES}

L'enseignement universitaire de théologie se présente de manière particulière en Alsace et en Moselle au regard de deux catégories de règles, souvent invoquées contre la mise en place d'un tel enseignement: la loi de 1905 n'a 
pas été introduite dans les départements recouvrés et l'article L. 141-6 du Code de l'éducation y fait l'objet d'une interprétation particulière. Dans les deux cas, on peut cependant s'interroger sur la spécificité juridique qui en résulte.

\subsubsection{NON-APPLICATION DE LA LOI DE 1905}

Un point ne fait pas de doute: la loi du 9 décembre 1905 concernant la séparation des Églises et de l'État n'est pas applicable en Alsace-Moselle. Quelles en sont les conséquences pour notre sujet? Cette loi ne parle aucunement de l'enseignement universitaire de la théologie. Sa non-application en Alsace et Moselle pourrait donc apparaître comme dépourvue de conséquence au regard du régime de la théologie universitaire.

Cependant, selon une interprétation assez bien répandue, l'enseignement de la théologie serait une forme de subventionnement du culte prohibé par l'article 2 de cette loi $^{1}$. D'ailleurs, en application de ce raisonnement, la faculté de théologie protestante de l'université de Paris a été supprimée après le vote de la loi du 9 décembre 1905 et transformée en institut privé. Précédemment, c'est aussi par le biais financier qu'ont été supprimées les facultés de théologie catholique ${ }^{2}$ existant en France. Ce dernier exemple illustre cependant l'ambiguité du sujet: si les crédits des facultés de théologie catholique ont été supprimés en 1885, ce n'est pas sur le fondement du principe de non-subventionnement instauré par la loi de 1905, celle-ci n'existant pas encore à ce moment, mais en raison d'une décision politique prenant place dans le conflit entre le gouvernement républicain et l'Église catholique. Dès lors, la suppression de la théologie protestante de l'université de Paris n'était-elle pas d'abord, elle aussi, une décision de nature politique, plutôt que la conséquence juridique nécessaire de l'adoption de l'article 2 de la loi de 1905?

Quoi qu'il en soit, l'interprétation actuelle de l'article 2 ne permet pas de conclure aisément à son incompatibilité avec le financement d'un enseignement de théologie au sein d'une université publique. Certes, on avance l'idée que l'enseignement de la théologie serait une forme de soutien par l'Université publique à la formation des clercs et que les fonds publics employés pour cet enseignement s'analyseraient en un soutien financier pour le fonctionnement

1. V. les observations de Francis Messner: «Formation des ministres du culte et des cadres religieux en Alsace-Moselle», JCl. Alsace-Moselle, 2017, fasc. 235, nº 32.

2. Loi de finances du 21 mars 1885 . 
des institutions religieuses bénéficiant de l'activité des personnes formées dans ce cadre. On peut d'ailleurs relever qu'une jurisprudence traditionnelle considère comme illégale l'aide financière apportée par des personnes publiques en vue de la formation religieuse ${ }^{3}$.

Mais ce raisonnement a toujours été bancal car, à le prendre à la lettre, toute formation universitaire profitant à du personnel religieux ou à de futurs personnels religieux devrait dans cette logique être considérée comme un soutien illégal au profit des institutions religieuses et, par suite, l'université devrait être interdite aux clercs et futurs clercs. Une telle approche serait cependant constitutive d'une discrimination fondée sur les convictions. Or, dès le début $\mathrm{du} x \mathrm{x}^{\mathrm{e}}$ siècle, la jurisprudence s'est refusée à admettre que le principe de laïcité impliquait l'exclusion des clercs de l'université ${ }^{4}$.

Dans la période récente, la jurisprudence a évolué concernant le financement public d'activités qui n'ont pas en elles-mêmes un caractère cultuel. Tel est le cas pour un enseignement universitaire, même s'il porte sur un sujet religieux. L'étude de la théologie n'est pas par elle-même une activité religieuse ou cultuelle ni une formation «professionnelle» destinée aux cultes. Or, le seul fait qu'une activité non cultuelle puisse comporter un avantage pour une institution cultuelle ne suffit plus dans le cadre de la jurisprudence actuelle pour la considérer comme relevant de l'interdiction de financement public défini par l'article 2 de la loi de 1905. La jurisprudence s'est affirmée notamment au sujet du financement d'objets ou d'équipements culturels pouvant aussi servir à des fins cultuelles, tels que par exemple un instrument de musique ${ }^{5}$. Il résulte désormais de cette jurisprudence que dès lors qu'il existe un intérêt général suffisant pour qu'un financement public soit attribué, le fait que son objet puisse également profiter au culte ne rend pas ce financement contraire à l'article 2 de la loi du 9 décembre 1905. Le même raisonnement peut s'appliquer au financement public d'enseignements. Si ces enseignements sont conformes à un intérêt général, leur financement public est légal. C'est ainsi que la jurisprudence récente admet que des activités d'enseignement et de recherche concernant la doctrine et la culture chrétienne ou encore des activités de réflexion spirituelle ou doctrinale ne tombent pas

3. CE, 13 mars 1953, Ville de Saumur: Lebon, p. 131: bourse municipale pour un étudiant pour suivre une formation de prêtre; 2 avr. 1992, n 102486, Tusques et Marcaillou: subvention à une direction diocésaine de l'enseignement.

4. CE, 10 mai 1912, Abbé Bouteyre: Lebon, p. 561. Dans ses conclusions, le commissaire du gouvernement Helbronner soulignait «qu'un évêque, un rabbin, enseignent dans des chaires publiques, à Paris, et un éminent prélat dirige aujourd'hui l'académie de France à Rome».

5. CE, ass., 19 juill. 2011, no 308544, Commune de Trélazé: RFDA 2011, p. 982. 
sous le coup de l'article 2 de la loi de $1905^{6}$. De façon générale, les activités d'études ne sont pas concernées par l'interdiction de subventionnement ${ }^{7}$. On relèvera que, sur un autre plan, la reconnaissance de diplômes ayant la théologie pour objet n'est pas contraire à l'article 2 de la loi de $1905^{8}$.

Pour ces différents motifs, on peut s'interroger si la possibilité légale de financer des enseignements théologiques correspond vraiment à une particularité du droit local alsacien mosellan du fait de la non-introduction de la loi de 1905 dans les départements recouvrés, ou si, en droit, un tel financement n'est pas susceptible d'être légalement mis en œuvre sur l'ensemble du territoire national dès lors qu'il présente un caractère d'intérêt général. Or, dans la période récente, l'intérêt général qui s'attache à doter le personnel religieux d'une formation appropriée, y compris universitaire, a été clairement reconnu. Les positions restrictives qui voudraient exclure une telle formation du financement public se font certes encore entendre, mais ne correspondent plus à l'état de la jurisprudence relative à l'interprétation de la loi de 1905.

Inversement, ne faudrait-il pas considérer que si l'on pouvait refuser à bon droit tout intérêt public à l'enseignement de la théologie, ce défaut d'intérêt public devrait être opposé à un tel enseignement même en AlsaceMoselle, indépendamment de la non-application de la loi de 1905? Car indépendamment de cette loi, tout financement public doit être motivé par l'intérêt général ${ }^{9}$.

En tout état de cause, si l'argument de l'article 2 ne peut être opposé à un enseignement de théologie dans l'université publique en Alsace-Moselle, ceci vaut non seulement pour la théologie catholique et protestante, mais pour tous les types de théologie, l'exclusion de la loi de 1905 valant pour toutes les formes d'expression religieuse et non seulement pour les cultes dits «statutaires», c'est-à-dire faisant l'objet en droit local d'un statut spécifique $^{10}$.

6. CAA Lyon, 2 févr. 2003, no 00LY00905, Bougaud et a.: AJDA 2006, p. 821, concl. D. BESLE; CAA Nantes, 31 juill. 2002, $\mathrm{n}^{\circ}$ 02NT010145, Région de Bretagne.

7. CE, $1^{\text {er }}$ juin 1956, Canivez: RFDA 1956, p. 253.

8. CE, 9 juill. 2010, n $\mathrm{n}^{\circ}$ 327663, Fédération nationale de la libre pensée: Lebon, p. 268.

9. CAA Nancy, 6 mars 2008, n ${ }^{\circ}$ 07NC00083, Ville de Soultz.

10. Il s'agit du culte catholique, du culte israélite et des cultes protestants réformés et de la Confession d'Augsbourg. 


\subsubsection{NON-APPLICATION DE L'ARTICLE L. 141-6 DU CODE DE L'ÉDUCATION EN TANT QU'IL S'OPPOSERAIT AU MAINTIEN DES FACULTÉS DE THÉOLOGIE}

Aux termes de l'article L. 141-6 du Code de l'éducation:

«Le service public de l'enseignement supérieur est laique et indépendant de toute emprise politique, économique, religieuse ou idéologique; il tend à l'objectivité du savoir; il respecte la diversité des opinions. Il doit garantir à l'enseignement et à la recherche leurs possibilités de libre développement scientifique, créateur et critique.»

La question soulevée par cet article est de savoir si cette définition du service public de l'enseignement universitaire exclut qu'un enseignement de théologie y soit dispensé. On analysera sur le fond cette problématique dans la deuxième partie de la présente étude, en soulevant pour l'immédiat la seule question de la portée de cette disposition en Alsace et en Moselle. La question est de savoir si cette disposition peut être comprise comme mettant en cause la législation locale.

Le problème a été abordé à l'occasion d'un recours formé contre le décret n 2011-1169 du 22 septembre 2011 portant création de l'université de Lorraine en y intégrant l'université de Metz, laquelle dispense des enseignements de théologie et délivre des diplômes dans cette matière ${ }^{11}$. Implicitement, le rapporteur public Rémi Keller retient le caractère non applicable de cet article en Alsace-Moselle en tant qu'il pourrait être compris comme s'opposant à un enseignement de théologie puisque, après avoir affirmé que la dispense des enseignements de théologie et la délivrance de diplômes dans cette matière « est légale en raison de la législation spéciale, issue du concordat, en vigueur dans les départements d'Alsace et de Moselle», il se borne, pour écarter la violation de cette disposition, à relever que le décret ne prévoit pas, par lui-même, «que des enseignements à caractère religieux pourraient être dispensés hors de l'Alsace et de la Moselle». Quant à la circonstance que les diplômes à caractère religieux seront désormais délivrés par l'université de Lorraine, dont le siège est à Nancy, en Meurthe-et-Moselle, «elle n'est pas par elle-même contraire au principe de laïcité». Ces justifications sont quelque peu approximatives, ${ }^{12}$ mais écartent bien l'article L. 146-1 comme

11. V. infra 1.2.2. les développements relatifs au Centre autonome d'enseignement de pédagogie religieuse de Metz.

12. CE, 23 juin 2014, no 354198, Union nationale des étudiants de France (UNEF) et a. Le Conseil d'État relève que le décret attaqué «n'a ni pour objet ni pour effet de transférer dans des établissements situés en dehors du territoire des départements du Bas-Rhin, du Haut-Rhin et de la Moselle les enseignements à caractère religieux dispensés par 
moyen de contestation de la présence d'enseignements de théologie dans la nouvelle université.

L'article L. 141-6 du Code de l'éducation est issu de l'article 3 de la loi no 84-52 du 26 janvier 1984 sur l'enseignement supérieur. Il n'est pas douteux qu'au moment de l'adoption de cette loi le législateur n'avait pas l'intention de remettre en cause l'enseignement de la théologie dans les universités d'Alsace et de Moselle. En vertu de cette intention du législateur, l'application de cet article ne peut être interprétée en Alsace et Moselle comme s'opposant à un enseignement de théologie. On peut encore se référer à une autre base, à savoir l'article L. 481-1 du Code de l'éducation. Cet article se borne il est vrai à prévoir que les dispositions particulières aux départements de l'Est régissant l'enseignement y demeurent en vigueur. Cependant, sur recours des autorités religieuses qui craignaient que l'on fasse valoir à cette occasion l'abrogation des dispositions ne relevant pas du livre IV «Établissements d'enseignement scolaire» dont fait partie l'article L. 481-1, le Conseil d'État a estimé que l'intégralité du droit local de l'enseignement était maintenue, la codification devant intervenir à droit constant ${ }^{13}$. Or l'enseignement de la théologie est un des éléments constitutifs du droit local de l'enseignement. En d'autres termes, l'adoption du Code de l'éducation, notamment de l'article L. 141-6, ne peut être opposée à l'enseignement de la théologie dans les universités d'Alsace et de Moselle.

\subsection{DISPOSITIONS PARTICULIÈRES DE «DROIT LOCAL » ORGANISANT L'ENSEIGNEMENT UNIVERSITAIRE DE LA THÉOLOGIE}

Le droit local se définit non seulement par l'absence d'application de certaines dispositions de droit commun comme la loi de 1905, mais aussi par le maintien des dispositions en vigueur au moment du retour de l'Alsace et de la Lorraine sous la souveraineté française et par l'adoption après 1918 par les autorités françaises de dispositions particulières à ces territoires.

l'université de Metz» et que la circonstance que les diplômes sanctionnant ces enseignements seront délivrés par l'université de Lorraine dont le siège est à Nancy n'est pas de nature à méconnaître les dispositions de l'article 2 de la loi du 9 décembre 1905 ou à porter atteinte au principe de neutralité religieuse de l'enseignement supérieur public rappelé notamment par l'article L. 141-6 du Code de l'éducation.

13. CE, 6 juin 2001, nos 224053, 224138, 224145, 224255, 224326, Archevêque de Strasbourg et a. : RDL, $\mathrm{n}^{\circ} 33,2001$, p. 52. 


\subsubsection{LE MAINTIEN DE CERTAINS ÉLÉMENTS DU DROIT APPLICABLE AU MOMENT LA RÉINTÉGRATION DANS LA SOUVERAINETÉ FRANÇAISE}

Aux termes de l'article 3 de la loi du 19 octobre 1919, «les territoires d'Alsace et de Lorraine continuent, jusqu'à ce qu'il ait été procédé à l'introduction des lois françaises, à être régis par les dispositions législatives et réglementaires qui y sont actuellement en vigueur». Il est souvent admis qu'en vertu de cette loi, au moment du retour de l'Alsace et de la Lorraine à la France, les dispositions locales relatives à l'enseignement universitaire de la théologie sont restées en vigueur. Cette analyse paraît trop simpliste sinon fausse. En effet, dès l'arrivée des troupes françaises à Strasbourg fin 1918, l'université allemande a été complètement balayée dans son personnel comme dans sa base juridique. Des dispositions antérieures à 1918, il ne subsiste que la convention de 1902.

\section{Suppression du régime universitaire allemand remplacé immédiatement par les règles françaises}

L'université allemande a cessé de fonctionner dès le 7 décembre 1918. Les cours ont repris quelques semaines après. L'Alsace-Lorraine n'étant pas officiellement réintégrée dans la souveraineté française, les règles allemandes relatives au fonctionnement de l'université (y compris en matière d'enseignement de la théologie) auraient dû continuer à s'appliquer. En réalité, c'est une nouvelle université qui est inaugurée le 22 novembre 1919, après qu'un décret du Président de la République du 19 novembre 1919, visant les lois françaises relatives à l'organisation universitaire, a créé des chaires dans les différentes facultés, un arrêté du 12 avril ayant procédé à la nomination des professeurs (9 et 11 pour les deux facultés de théologie). Dès la réouverture de l'université, on a procédé de fait à l'application, chaque fois que cela était possible, des règles propres aux universités françaises.

Par la suite, par l'adoption du décret du 30 mai 1924, l'essentiel du droit français de l'enseignement supérieur a été introduit de façon formelle. Cette introduction est intervenue non par une clause générale, mais au moyen d'une énumération des textes rendus applicables, ce choix ayant été retenu notamment en raison de la problématique posée par la non-introduction du régime de séparation et du maintien des deux facultés de théologie.

La démarche retenue a été de rétablir ces deux facultés malgré l'avis contraire d'un certain nombre d'autorités et de les intégrer autant que possible dans le cadre «normal» de l'université française et de limiter autant qu'il se pouvait le caractère spécifique de ces institutions afin d'en minimiser l'originalité. Aussi, l'article 4 du décret du 30 mai 1924 les traite-t-il au même 
titre que les autres composantes de l'université: «L'université de Strasbourg se compose des facultés de théologie catholique, de théologie protestante, de droit et des sciences politiques, de médecine, des sciences, des lettres et de pharmacie.»

Le décret du 30 mai 1924 ne comporte que quelques dispositions particulières pour ces deux facultés de théologie:

- l'article 5, s'agissant des conditions de nomination et d'avancement, et à titre transitoire et par dérogation à l'article 6 du décret du 22 août 1854 , prévoit que le doctorat en théologie n'est pas exigé des candidats aux chaires magistrales pendant une période de douze ans. Les promotions de classe sont faites sur la proposition de deux commissions siégeant à Strasbourg, présidées par le recteur, une pour chaque faculté;

- l'article 7 désigne nommément les huit chaires de la faculté de théologie catholique et les six chaires de la faculté de théologie protestante;

- l'article 6 prévoit qu'en cas de conflit entre un membre des facultés de théologie et l'autorité ecclésiastique, il sera fait application de l'article 40 du décret du 21 juillet 1897 relatif au régime scolaire et disciplinaire des universités. Cet article prévoit comme mesures possibles la suspension d'un cours par le recteur ou la fermeture temporaire d'une faculté ou de certains enseignements par le ministre.

Par la suite, les transformations juridiques de l'université de Strasbourg vont confirmer le caractère de droit commun de ces facultés ${ }^{14}$, qui ne présentent des règles d'organisation spécifiques que sur des points de détail, excepté bien sûr la convention de 1902, applicable à la seule faculté de théologie catholique ${ }^{15}$.

14. À la suite de l'éclatement de l'université unique en 1970 et de la création de l'Université des sciences humaines, devenue Université Marc Bloch, les facultés ont pris le statut d'instituts d'université en 1984, ce qui leur a garanti une certaine autonomie. La nouvelle loi «Libertés et responsabilités des universités» (LRU) du 10 août 2007 ne remet pas en cause le statut des instituts à caractère dérogatoire. Le regroupement des trois universités strasbourgeoises aboutit au premier janvier 2009. Les facultés retrouvent leur nom, tout en gardant le même statut d'autonomie au sein de l'université de Strasbourg.

15. C'est à tort que certains auteurs ont laissé entendre que des dispositions locales relatives à l'université et prévoyant un avis des autorités cultuelles protestantes avant la nomination des professeurs de théologie protestante seraient restées en vigueur après 1919. Jusqu'à sa modification par le décret du 18 avril 2006, l'article 11 du décret du 26 mars 1852 portant réorganisation des cultes protestants comportait une disposition prévoyant que le directoire de l'Église de la Confession d'Augsbourg donne son avis motivé sur les candidats aux chaires de la faculté de théologie. Cette disposition visait les chaires de théologie de l'ancien séminaire protestant. Mais la pratique s'est maintenue durant la période du Reichsland sur la base du règlement de l'université et sans doute aussi pendant quelque temps après 1919. 


\section{La convention du 5 décembre 1902}

Ce document ${ }^{16}$ est en fin de compte la seule disposition de droit local applicable à l'enseignement universitaire de théologie datant d'avant 1918 et maintenu en vigueur après cette date ${ }^{17}$. Il s'agit d'un élément d'importance au plan juridique et pratique.

Cette convention conclue entre le gouvernement allemand et le Saint-Siège n'est pas un document unique, mais comme le dit Francis Messner ${ }^{18}$ représente tout un bloc de textes juridiques ${ }^{19}$ : la convention elle-même, portant création d'une faculté de théologie catholique, les statuts des facultés de théologie de Bonn et de Breslau auxquels renvoie la convention, une note explicative du baron Georg von Hertling du 20 novembre 1902, le bref cum venerabilis du 3 septembre 1903 qui accorde à la faculté le droit de conférer le grade de licencié et de décerner le titre de docteur.

Le maintien en vigueur de cet accord résulte d'un échange de lettres entre le gouvernement français et le Saint-Siège du 17 novembre $1923^{20}$. La nature juridique de la convention de $1902^{21}$ a pu faire l'objet de discussions ${ }^{22}$ dans

16. L'accord a été publié par une ordonnance no 1065 du 9 août 1903: Gesetzblatt für ElsassLothringen 1903, p. 57.

17. Pour autant que l'on accepte de considérer qu'il n'y a pas de rupture de base juridique entre cette convention telle qu'elle s'appliquait jusqu'en 1918 et celle remise en vigueur par l'échange de lettres de 1923 (V. infra).

18. F. MESSNER, «Formation des ministres du culte et des cadres religieux en Alsace-Moselle», art. cit., $\mathrm{n}^{\circ} 28$.

19. L'ensemble des textes juridiques relatifs aux facultés de théologie de Strasbourg (18521978) et au Centre de Metz est publié dans J. Schlick (dir.), Églises et État en Alsace et en Moselle, Strasbourg, Cerdic, 1979, p. 301-316.

20. Par cet échange de lettres, l'État français accepte de se substituer dans cet accord international à l'État allemand. On peut se demander si cette substitution vaut novation et si par suite il y a nouvel accord, ou si c'est l'accord ancien qui reste en vigueur sans solution de continuité. Au regard des termes de l'échange de lettres, cette deuxième solution semble devoir être retenue: "il a été reconnu que la convention du 5 décembre 1902 conserve sa valeur sous le régime français». Mais dans une lettre du 16 novembre 1923 de la nonciature apostolique au ministre français des Affaires étrangères, il est dit que l'accord était devenu caduc en raison d'une déclaration du Saint-Siège du 15 mars 1919 et que la remise en vigueur a été demandée par le gouvernement français. À cette occasion, le contenu et la portée de la Convention ont été précisés et pour partie modifiés (les autorités religieuses renoncent à l'engagement du gouvernement allemand de nommer à la faculté de lettres un enseignant d'histoire et un enseignant de philosophie de religion catholique).

21. V. É. SANDER, «De 1902 à 2002: la pérennité du statut de la faculté de théologie catholique », Rev. des sciences religieuses, $\mathrm{n}^{\circ} 78-1,2004$, p. 11.

22. On lui a parfois attribué le caractère d'acte unilatéral, soit du Saint-Siège, soit de l'État, sous prétexte que les deux signataires sont des personnes morales d'essence différente, ce qui s'opposerait à ce qu'ils puissent nouer des liens contractuels. 
le passé. Aujourd'hui, son caractère de véritable accord international n'est plus véritablement contesté, la capacité du Saint-Siège comme de l'autorité étatique de conclure de tels accords étant admise. Ce caractère d'accord international a été reconnu ${ }^{23}$ par les autorités allemandes avant 1918. L'échange de lettres qui le maintient l'admet aussi implicitement ${ }^{24}$. La doctrine va dans le même sens par analogie au concordat de $1801^{25}$.

La convention en question lie les parties jusqu'à dénonciation par l'une d'entre-elles. Se pose la question de ses effets par rapport aux tiers. En application de l'article 55 de la Constitution, «les traités ou accords régulièrement ratifiés ou approuvés ont dès leur publication une autorité supérieure à celle des lois sous réserve pour chaque accord de son application par l'autre partie». Cette disposition peut-elle s'appliquer à la convention de 1902 ? Celle-ci a certes fait l'objet d'une publication durant la période allemande. Mais ce n'est pas le cas pour l'échange de lettres de confirmation de 1923. Une telle confirmation doit-elle, elle aussi, être publiée pour que la convention ait une autorité supérieure aux lois? La question n'a pas été définitivement tranchée. On verra plus loin la question de la conformité de la convention avec le principe constitutionnel de laïcité.

L'objet de la convention correspond à un engagement réciproque, celui des autorités religieuses de former les clercs dans le cadre de la faculté de théologie, celui des autorités étatiques de fournir des garanties quant à l'orthodoxie des enseignants nommés. Dans ce cadre, les autorités catholiques acceptent que «l'instruction scientifique sera donnée aux jeunes clercs du diocèse de Strasbourg par une faculté de théologie catholique érigée à l'Université de Strasbourg ${ }^{26} »$. En contrepartie, l'évêque a le droit de refuser la nomination ou le maintien d'un enseignant dont l'enseignement ou la conduite heurte la doctrine catholique ${ }^{27}$. En outre, aux termes des statuts

23. Il s'agit d'une convention internationale d'un type particulier en tant qu'elle porte non sur des rapports interétatiques, mais sur l'organisation dans une institution étatique d'un enseignement de la théologie catholique.

24. F. Messner, P.-H. Prélot et J.-M. Woehrling (dir.), Traité de droit français des religions, Paris, Litec, 2003, n 2918.

25. Ainsi, Jules Regula mentionne en 1938 la convention de 1902 parmi les conventions internationales intéressant l'Alsace et la Lorraine (Le droit applicable en Alsace et en Lorraine, Paris, Dalloz, 1938, p. 699).

26. Art. 1 de la convention.

27. Les exigences à l'égard du personnel enseignant concernant leur «orthodoxie» sont très étendues: l'article 2 de la convention prévoit que la nomination se fera après entente préalable avec l'évêque et que les enseignants ont à faire une profession de foi entre les mains du doyen suivant les formes et règles de l'Église (art. 3). Si la preuve est fournie par l'autorité ecclésiastique qu'un professeur doit être considéré incapable d'assurer son 
des facultés de théologie de Bonn et de Breslau, les livres et les programmes des cours seront soumis à l'évêque. L'article 2 de la convention précise les diverses branches de l'enseignement offert par la faculté.

On retrouve des préoccupations similaires à celles fondant le concordat de 1801 : l'accord n'a pas pour objet de favoriser une religion déterminée en contrariété avec la neutralité religieuse de l'État, mais d'obtenir un acquiescement sur certaines modalités de formation en échange duquel certaines garanties sont concédées. Comme tout compromis, la convention exprime un équilibre délicat et suppose, pour pouvoir fonctionner, une retenue prudente de la part de chaque partie. En soi, l'obligation faite à des enseignants de respecter une certaine orthodoxie et de pratiquer un «devoir de réserve " n'est pas incompatible avec l'indépendance d'esprit qui doit caractériser le travail universitaire. On pourrait trouver en dehors de la théologie des situations où la liberté universitaire doit tenir compte d'un certain ordre intellectuel ${ }^{28}$. La fragilité du système mis en place par la convention tient au fait qu'elle est plaquée sur le droit universitaire français, pratiquement sans aucun élément d'ajustement de nature à concilier en cas de difficulté les règles de l'ordre universitaire et les principes de l'ordre catholique ${ }^{29}$. Mais peut-être est-ce justement cette absence de mécanisme de résolution des difficultés qui a contraint les deux parties à éviter un conflit, lequel aurait inévitablement conduit à la rupture de l'accord.

Dans la pratique, aucun enseignant n'a été révoqué depuis 1919 (ni d'ailleurs avant) alors que certains enseignants ont fait l'objet de condamnations par la curie romaine. Sans doute les exigences d'orthodoxie sont-elles comprises aujourd'hui autrement qu'au début du $\mathrm{xx}^{\mathrm{e}}$ siècle. Par ailleurs, les

enseignement en raison d'un manque d'orthodoxie ou d'un manque de conduite au regard des règles de vie d'un prêtre, le Gouvernement pourvoira sans délai à son remplacement (art. 5).

28. Chaque matière universitaire a des postulats dont la remise en cause est difficilement acceptable. Ainsi, un enseignement juridique qui réfuterait toute pertinence à l'État de droit ou un enseignement de l'Histoire fondé sur le négationnisme seraient considérés comme une violation des principes de l'approche universitaire.

29. Un protocole signé en 1979 entre l'archevêque de Strasbourg et la faculté de théologie catholique indique la procédure que les deux parties se sont engagées à respecter lors de la vacance d'un poste: le doyen informe l'évêque de la vacance et lui communique la liste des candidats retenus par les jurys compétents (commission spéciale consultative pour la qualification et comité de sélection pour le recrutement). L'évêque fait connaître sa position et celle du Saint-Siège avant que les dossiers ne soient transmis au ministère pour nomination. Le protocole prend en compte également la distinction entre prêtres et laïcs. Ce protocole n'a pas de valeur juridique bien définie. Il peut être considéré comme une interprétation acceptée par les parties des conditions de mise en œuvre de la convention. 
enseignants auxquels des reproches pourraient être faits préfèrent solliciter une affectation dans une autre unité de formation et de recherche. Il en résulte un hiatus entre un texte conventionnel très rigide et une pratique évolutive.

\subsubsection{L'ÉVOLUTION DU DROIT LOCAL DE L'ENSEIGNEMENT UNIVERSITAIRE APRÈS LE RETOUR À LA FRANCE}

Si le droit local de l'enseignement universitaire de la théologie comporte peu de dispositions applicables avant 1918 et maintenues en vigueur, en dehors de la convention de 1902, il a par contre connu des évolutions du fait des autorités françaises. On distinguera entre des modifications réalisées et d'autres qui ont échoué.

Une «extension réussie»: la création du Centre autonome d'enseignement de pédagogie religieuse (CAEPR) de Metz

La convention du 25 mai 1974 entre le Saint-Siège et la République française relative au Centre autonome d'enseignement de pédagogie religieuse de l'université de Lorraine apparaît comme un prolongement de la convention de 1902 à laquelle ce texte renvoie pour tout ce qui concerne les rapports entre le Centre et les autorités religieuses.

La circonstance que, plus de 55 ans après le retour de l'Alsace-Moselle à la France, le droit local de l'enseignement de théologie connaisse un nouveau développement a une forte signification tant juridique que symbolique: l'enseignement de la théologie à l'université n'est pas un régime transitoire en sursis dans l'attente d'une disparition.

Le Centre autonome d'enseignement de pédagogie religieuse a été créé pour répondre au besoin de formation des enseignants appelés à intervenir dans le cadre de l'enseignement religieux dans l'enseignement secondaire, au moment où la scolarité était prolongée jusqu'à l'âge de 16 ans. Une première solution a consisté à ouvrir, en 1965, à Metz, un Centre de pédagogie religieuse constituant en une extension de la faculté de théologie de Strasbourg. Le rattachement de la Moselle à l'académie Nancy-Metz et la création d'une université à Metz ont conduit à la mise en place d'une structure propre ${ }^{30}$ qui propose une formation longue et une formation courte avec

30. Le Centre a été rattaché statutairement à l'UFR de sciences humaines et arts alors que les autorités ecclésiastiques souhaitaient qu'il soit doté d'un statut d'institut autonome. Sur ce sujet, V. Rép. min. nº 00739: JOAN, 18 juill. 2002, p. 1614, J.-L. Masson. 
des enseignements spécifiquement théologiques et bibliques, mais aussi de l'anthropologie religieuse, de l'histoire, de la philosophie, de la patristique, de la spiritualité et de l'éthique.

Ce centre a proposé ensuite un cursus d'études théologiques et pédagogiques complet, conformément aux schémas de l'université française, à un public assez large de personnes qui avaient le souci d'acquérir une solide formation théologique ou qui souhaitaient l'approfondir et trouver des éléments de réponse face aux problématiques contemporaines. Il s'est aussi ouvert à la recherche. Pendant des années, les cours ont été assurés dans les locaux du grand séminaire par les enseignants de l'université de Lorraine à Metz ${ }^{31}$.

En 2018, l'évêque de Metz a décidé de ne plus recourir à ce centre pour former les séminaristes et les enseignants de religion à l'école ${ }^{32}$. Un «Institut Saint-Nicolas » a été créé dans le cadre duquel les quatre diocèses de Lorraine (Metz, Nancy, Saint-Dié et Verdun) proposent une formation en théologie axée sur les éléments fondamentaux de la foi chrétienne et orientée vers l'exercice d'une mission dans l'Église catholique.

De son côté, le CAEPR, devenu département de théologie de l'université de Lorraine, a décidé, compte tenu de la nouvelle situation et de la rupture des liens de subordination avec le catholicisme, d'élaborer une nouvelle offre de formation ouverte sur les trois monothéismes historiques. Une chaire est créée en théologie islamique, venant compléter l'expertise déjà présente sur le christianisme. Désormais, l'enseignement et la recherche du département de théologie se veulent multiconfessionnels et en toute liberté par rapport aux institutions religieuses. Il ne s'agit pas d'un département qui enseigne les sciences des religions, mais qui continue de faire œuvre théologique. Aujourd'hui, ce département de théologie se présente comme la seule institution en France à proposer une «formation publique de théologie sans affiliation confessionnelle ${ }^{33} »$.

31. Sur la contestation suscitée par l'intégration du Centre dans l'université de Lorraine, V. supra note 12.

32. Pour Jean-Christophe Meyer, vicaire général de Metz, «les enseignements de cette formation étaient incomplets et ne développaient pas assez les fondements de la théologie dogmatique» (La Vie, 22 juin 2018). Après un audit dirigé par l'évêque de Metz, JeanChristophe Lagleize, le diocèse et l'équipe du CAEPR avaient travaillé conjointement sur de nouvelles offres de formation. Cet effort d'ajustement n'a semble-t-il pas abouti.

33. http://e-theologie.fr/ [consulté le 7 janv. 2020]. 
Du côté des autorités religieuses, on évoque une possible demande de l'évêque de Metz au Vatican de procéder à la dénonciation de la convention de 1974.

\section{Un «échec d'extension»: la création d'une faculté de théologie musulmane}

Dès $1968^{34}$, on a pensé qu'il serait possible de valoriser le potentiel «théologie, sciences religieuses et histoire des religions » existant à Strasbourg pour créer une faculté de théologie musulmane au sein de l'Université des sciences humaines de Strasbourg ${ }^{35}$. En 1996, le président de l'USHS, Albert Hamm, a demandé au professeur Étienne Trocmé, ancien doyen de la faculté de théologie protestante de rédiger un rapport au sujet du développement des sciences des religions à l'USHS dans le cadre du projet d'établissement. Dans son rapport, celui-ci évoque notamment l'intérêt de former à l'islam les cadres intellectuels pour favoriser l'élaboration d'une pensée musulmane adaptée à la situation française dans des lieux de connaissance autonomes. Étienne Trocmé préconise la création, au sein de l'USHS, d'un Institut de théologie musulmane dont l'objectif serait essentiellement de former des savants, des théologiens universitaires. L'idée de l'instauration d'un département de formation pratique des imams est écartée. Étienne Trocmé promeut de fait l'hypothèse d'une théologie musulmane non confessionnelle proche du modèle de la théologie protestante. En raison d'oppositions politiques, de tensions intra-universitaires et des craintes exprimées par certains dignitaires religieux notamment catholiques, ainsi que d'une absence de détermination des autorités publiques, les préconisations du rapport Trocmé n'ont pas été suivies d'effet. L'idée avait pourtant connu des soutiens répétés ${ }^{36}$.

Ce projet de développement d'un enseignement de théologie musulmane est à replacer dans les politiques de formation des cadres religieux musulmans

34. À l'occasion de l'application de la loi Faure du 12 novembre 1968 sur l'orientation de l'enseignement supérieur, certains avaient imaginé d'ériger à Strasbourg une université des religions comportant les deux facultés historiques de théologie, une composante spécialisée en histoire des religions et une faculté de théologie musulmane qui aurait été créée à cette occasion: F. MESSNER, «Les pouvoirs publics et la formation des cadres religieux», in F. MESSNER, A.-L. Zwilling (dir.), Formation des cadres religieux en France. Une affaire d'État?, Genève, Labor et Fides, 2010, p. 20.

35. Il y eut aussi quelques initiatives locales, comme la création du Groupe d'études et de recherches islamologiques (GERI) à la faculté de théologie protestante de l'université de Strasbourg, en 1981.

36. Les rapports Baroin (Pour une nouvelle laïcité, 2003) et Machelon (Les relations des cultes avec les pouvoirs publics, 2006) ont préconisé la création d'une faculté de théologie musulmane à Strasbourg. 
dont l'importance n'a cessé de croître ${ }^{37}$. La création d'un enseignement de théologie musulmane à Strasbourg aurait pu constituer un instrument important dans ce sens.

Il faut retenir de cette tentative qu'elle s'est appuyée davantage sur un contexte intellectuel que juridique: c'est la présence à Strasbourg d'une activité universitaire significative consacrée à la théologie qui a donné naissance au projet et non le statut juridique particulier des trois départements, si ce n'est que celui-ci permettait d'écarter aisément l'objection tirée de l'article 2 de la loi de 1905.

De l'examen qui précède, on peut conclure que les deux seules particularités juridiques existant en droit local pour l'enseignement universitaire de la théologie résident dans la non-application de la loi de 1905 et dans les conventions de 1902 et 1974, l'organisation des deux facultés de théologie relevant pour le reste du droit commun. Si l'on considère que l'article 2 de la loi de 1905 ne constitue pas un obstacle à l'enseignement de la théologie, que la convention de 1902 ne concerne que la seule théologie catholique et que la convention de 1974 a cessé de s'appliquer de facto, le bilan, sur le plan du cadre juridique, de la spécificité de l'enseignement de la théologie en Alsace et Moselle peut paraître limité. En va-t-il différemment si l'on se place au plan du droit constitutionnel?

\section{ENSEIGNEMENT DE LA THÉOLOGIE À L'UNIVERSITÉ PUBLIQUE ET PRINCIPES CONSTITUTIONNELS}

L'existence d'un enseignement de théologie à l'Université publique doit être compatible avec la Constitution. Cet impératif s'applique aussi pour les enseignements organisés en Alsace et Moselle. Toutefois, pour ces territoires, la question se dédouble: il convient de s'interroger sur les exigences constitutionnelles en matière d'enseignement universitaire de la théologie, mais il faut aussi examiner les aménagements spécifiques que l'ordre constitutionnel réserve à l'Alsace et à la Moselle. La deuxième question ne se pose que si les spécificités alsaciennes et mosellanes heurtent l'ordre constitutionnel de droit commun.

37. F. Messner, La formation des cadres religieux musulmans. Rapport au ministère de l'Éducation nationale, de l'Enseignement supérieur et de la Recherche et au ministère de l'Intérieur, 2015. 


\subsection{LE DROIT COMMUN DES EXIGENCES CONSTITUTIONNELLES EN MATIÈRE D'ENSEIGNEMENT UNIVERSITAIRE DE LA THÉOLOGIE}

À l'évidence, la Constitution ne traite pas explicitement ou de manière directe la question d'un enseignement de théologie à l'Université publique. Cette question se pose à travers le prisme du principe constitutionnel de laïcité affirmé dans l'article $1^{\text {er }}$ de la Constitution ${ }^{38}$, mais également dans son préambule ${ }^{39}$ et comme principe fondamental reconnu par les lois de la République $^{40}$. Le principe constitutionnel de laïcité doit se distinguer de celui figurant explicitement ou implicitement dans des textes législatifs.

Sa portée a été précisée par la décision du Conseil constitutionnel du 23 février $2013^{41}$ : «il en résulte la neutralité de l'État; il en résulte également que la République ne reconnaît aucun culte; le principe de laïcité impose notamment le respect de toutes les croyances, l'égalité de tous les citoyens devant la loi sans distinction de religion et que la République garantisse le libre exercice des cultes; il implique que celle-ci ne salarie aucun culte.» Sur la base de cette analyse, il nous faut répondre à trois questions.

\subsubsection{LA PRÉSENCE D'UN ENSEIGNEMENT DE THÉOLOGIE DANS UNE UNIVERSITÉ PUBLIQUE HEURTE-T-ELLE LE PRINCIPE CONSTITUTIONNEL DE LAÏCITÉ?}

L'enseignement à l'Université publique doit être laĩque. Le Conseil constitutionnel n'a pas encore précisé le contenu de cette exigence. L'article L. 141-6 susmentionné du Code de l'éducation a tenté une définition: "Le service public de l'enseignement supérieur est [...] indépendant de toute emprise politique, économique, religieuse ou idéologique; il tend à l'objectivité du savoir; il respecte la diversité des opinions. Il doit garantir à l'enseignement et à la recherche leurs possibilités de libre développement scientifique, créateur et critique.» Un enseignement de théologie peut-il correspondre à ces critères? L'indépendance de toute emprise, le libre développement critique,

38. «La France est une République indivisible, laique, démocratique et sociale.»

39. Point 13 du préambule de la Constitution du 27 octobre 1946: «L'organisation de l'enseignement public gratuit et laïque à tous les degrés est un devoir de l'État. »

40. CE, 6 avr. 2001, nos 219379, 221699, 221700, Syndicat national des enseignements de second degré (SNES): Lebon, p. 521.

41. Cons. const., 21 févr. 2013, n 2012-297 QPC, Association pour la promotion et l'expansion de la laïcité [Traitement des pasteurs des églises consistoriales dans les départements du Bas-Rhin, du Haut-Rhin et de la Moselle]: J.-M. WoenRLing, «Le droit local alsacienmosellan des cultes après les récentes décisions du Conseil constitutionnel»: RDP 2013, p. 532. 
le pluralisme d'opinion ne dépendent pas de la matière traitée, mais des conditions et garanties offertes par l'université. On peut cependant se poser la question de savoir si la théologie se prête à une démarche scientifique alors qu'elle est liée à des présupposés de croyance. Toutefois, les conceptions actuelles de la démarche «scientifique» mettent davantage l'accent sur les méthodes de travail que sur l'absence de présupposés subjectifs. Entre l'analyse littéraire ou le travail philosophique dont la place à l'université n'est pas contestée et la connaissance théologique, les parts respectives d'objectivité et de subjectivité ne sont pas nécessairement très différentes. L'opposition entre «sciences religieuses» et «théologie» initialement perçue comme de caractère structurel $^{42}$ s'est atténuée. Études bibliques, sciences des religions, sociologie religieuse, histoire des religions, exégèse et recherche théologique sont souvent combinées.

Dans le cas de l'université de Strasbourg, l'apport de la théologie à l'œuvre de formation et de recherche confiée aux établissements publics universitaires est clairement reconnu ${ }^{43}$. Ainsi, le rapport du Comité national d'évaluation de l'enseignement supérieur sur la faculté de théologie catholique de Strasbourg en 1991 admet que les axes d'enseignement et de recherche de la théologie trouvent leur place dans la recherche universitaire française y compris dans sa dimension laique. En dehors de la France, l'enseignement de la théologie au sein des universités européennes s'impose comme une tradition bien établie y compris dans les anciens pays communistes, ainsi qu'à travers la création de facultés ou de chaires de théologie musulmane. La théologie enseignée dans ce contexte se plie à la méthode universitaire et se combine avec d'autres disciplines (études des langues anciennes, droit des cultes, sciences sociales, philosophie, étude historico-critique des textes, etc.). Confronté à l'apport des autres savoirs, le théologien doit faire connaître les postulats de sa propre démarche ${ }^{44}$. En somme, la théologie en tant que discipline ou domaine académique constitue une dimension des sciences humaines et sociales. Au regard de la méthodologie mise en œuvre, le fait de vouloir exclure ce champ du savoir de la démarche universitaire pourrait être considéré comme marqué d'une partialité peu compatible avec les principes laïques. L'université est le lieu d'études de tous les savoirs. Écarter par

42. É. Poulat et O. Poulat, «Le développement institutionnel des sciences religieuses en France», ASSR, n 21, 1966, p. 23-36.

43. M. Deneken, «La théologie dans la nouvelle Université de Strasbourg», Recherches de science religieuse 2008/4, p. 527-546.

44. F. Messner, «Le statut des facultés de théologie en France», in S. Akgönul (dir.), Laïcité en débat, Strasbourg, Presses universitaires de Strasbourg, 2008, p. 255-276. 
principe un type de savoir particulier, au demeurant celui qui est à l'origine de sa création ${ }^{45}$, ne peut trouver de fondement constitutionnel et n'aurait pas d'équivalent au plan international ${ }^{46}$.

\subsubsection{LE FINANCEMENT PUBLIC D'UN ENSEIGNEMENT DE THÉOLOGIE DU FAIT DE SON INSERTION DANS LES PROGRAMMES D'UNIVERSITÉS PUBLIQUES EST-IL COMPATIBLE AVEC LE PRINCIPE CONSTITUTIONNEL DE LAÏCITÉ?}

Si le Conseil constitutionnel érige sur le plan des principes constitutionnels que «la République ne salarie aucun culte ${ }^{47}$ », il n'en a pas fait de même avec la règle figurant à l'article 2 de la loi de 1905 selon laquelle «la République ne subventionne aucun culte». Suivant en cela la jurisprudence du Conseil d'État ${ }^{48}$ et la doctrine dominante, le Conseil constitutionnel admet que le principe constitutionnel de laicité n'impose pas une telle interdiction de façon générale. Des motifs d'intérêt public peuvent justifier un financement public. La non-application de la loi de 1905 en Alsace et Moselle ne heurte donc pas un principe constitutionnel. Sans que le Conseil constitutionnel ait tranché ce point, on peut aussi considérer que le souci d'intégrer la réflexion théologique dans le cadre de l'Université publique et de favoriser la formation scientifique des personnels religieux peut constituer un motif d'intérêt public rendant compatible ce mode de financement avec le principe constitutionnel de laicité. Cette considération se trouve renforcée dans la période récente, dans laquelle l'action contre l'islamisme radical passe de plus en plus par un soutien public à la formation du personnel religieux musulman.

Dans l'esprit des autorités publiques, il est important que les responsables religieux puissent suivre une formation similaire à celle des autres cadres de la Nation. C'est dans cet esprit que la théologie figurait parmi les matières enseignées à l'Université impériale au début du XIX siècle. Le même esprit guide aujourd'hui les efforts déployés pour offrir aux cadres du culte musulman un enseignement universitaire leur permettant de développer une

45. La théologie figure parmi les enseignements initiaux des premières universités.

46. F. Curtit, A.-L. Zwilling, «L'enseignement de la théologie musulmane en Europe: contexte et contenu», in F. MESSNER, M. DENEKEn (dir.), La théologie à l'université: statut, programmes et évolutions, Genève, Labor et Fides, 2009, p. 151-171.

47. Il semble qu'il faille entendre par là une rémunération directe de ministres des cultes par une collectivité publique en dehors des emplois d'aumôniers dans des services publics ou pour des missions de service public autres que cultuelles. Un financement public d'une autre nature ne serait pas exclu.

48. CE, 16 mars 2005, n 265560, Ministère de l'outre-mer c. Gouvernement de la Polynésie française: AJDA 2005, p. 964 et p. 1463, note C. DuRAND-PRinborgne. 
réflexion théologique en rapport avec le contexte intellectuel, social et politique de la France. De nombreuses initiatives sont déjà engagées pour une meilleure approche universitaire de l'islam; il peut sembler artificiel d'en vouloir exclure la dimension théologique ${ }^{49}$.

\subsubsection{LA PARTICIPATION DES AUTORITÉS RELIGIEUSES À LA GESTION DES FACULTÉS DE THÉOLOGIE EST-ELLE CONSTITUTIONNELLE?}

Cette question est sans doute plus délicate et a été moins approfondie que les deux précédentes. On peut considérer à la fois qu'une collaboration est légitime, mais que certaines formes d'ingérence sont de nature à porter atteinte au principe d'indépendance des universités et des enseignantschercheurs, garanti par un principe fondamental reconnu par les lois de la République. Le travail universitaire est de moins en moins coupé des attentes de la société, que celles-ci concernent la formation professionnelle, le développement économique ou le débat de société. L'université doit être à l'écoute de ces attentes. On n'imagine pas qu'au motif d'indépendance elle ignore les attentes des secteurs professionnels dont elle est appelée à fournir les cadres. Dans le cas des études théologiques, le dialogue doit associer les institutions religieuses. Dans ce domaine comme dans d'autres, des conventions peuvent définir les termes d'une collaboration. Mais ces relations doivent certainement respecter l'autonomie respective des partenaires et plus particulièrement la liberté d'expression et de recherche des enseignants-chercheurs.

Le récent conflit survenu entre l'évêché de Metz et le centre de théologie de l'université de Metz illustre le fait que les deux parties risquent d'être perdantes d'une rupture de collaboration: l'université perd des étudiants et le lien avec l'objet de son étude; le diocèse perd le bénéfice de l'ouverture de ses futurs clercs à une recherche libre et pluridisciplinaire.

Dans cette discussion, tout reste question d'un équilibre que doivent garantir les modalités de mise en œuvre de la convention de 1902. Les termes mêmes des garanties exigées par l'institution ecclésiastique à l'égard des enseignants paraissent au regard des conceptions actuelles comme excessifs et incompatibles avec l'indépendance dont, en vertu des principes constitutionnels, doivent bénéficier les enseignants-chercheurs à l'université. Par contre, la pratique actuelle semble assez éloignée des termes de l'accord,

49. A.-L. Zwilling, «L'enseignement de l'islam dans les universités en France: une histoire mouvementée », in Droit et religion en Europe, Strasbourg, Presses universitaires de Strasbourg, 2014, p. 239-259. 
de sorte que malgré une activité universitaire très libre et exigeante, aucun conflit n'est survenu avec les autorités du diocèse de Strasbourg.

De l'analyse qui précède on peut conclure qu'il n'y a pas d'incompatibilité entre l'exercice de la théologie à l'Université publique et les principes du droit constitutionnel ${ }^{50}$ ni pour le droit local alsacien-mosellan, ni même pour le droit commun applicable dans le reste de la France. Cette problématique n'étant pas définitivement tranchée, notamment par la jurisprudence constitutionnelle, il paraît néanmoins justifié d'examiner les protections constitutionnelles spécifiques dont bénéficie le droit local alsacien-mosellan.

\subsection{LES GARANTIES CONSTITUTIONNELLES DE MAINTIEN DU DROIT LOCAL ET LES ÉTUDES THÉOLOGIQUES À L'UNIVERSITÉ PUBLIQUE}

Le droit local doit se conformer aux principes constitutionnels. Si donc, contrairement aux analyses qui précèdent, on devait arriver à la conclusion qu'une règle de valeur constitutionnelle, déduite du principe de laïcité ou du principe de l'indépendance des universités, s'oppose à l'existence d'un enseignement de théologie à l'Université publique, la circonstance que cet enseignement est prévu par le droit local ne suffit pas pour écarter l'incompatibilité avec la Constitution.

Toutefois, sans libérer le droit local de manière générale de l'obligation de conformité constitutionnelle, la jurisprudence constitutionnelle a institué des mécanismes de sauvegarde du droit local en cas de conflit avec le droit constitutionnel. La portée de ces mécanismes est limitée, mais elle peut trouver application, le cas échéant, dans le domaine de l'enseignement universitaire de la théologie. Ces mécanismes sont de deux ordres dont les caractéristiques sont assez semblables.

\subsubsection{LE DROIT LOCAL PROTÉGÉ PAR UN «PRINCIPE FONDAMENTAL RECONNU PAR LES LOIS DE LA RÉPUBLIQUE»}

En application de la jurisprudence constitutionnelle, la réitération par plusieurs textes de valeur législative de 1918 à nos jours du maintien en vigueur de dispositions du droit local a instauré une règle constitutionnelle selon laquelle, nonobstant leur possible incompatibilité avec le principe d'égalité devant la loi appliqué au plan territorial, les dispositions législatives et

50. Ce constat vaut a fortiori pour les principes du droit international applicables en France. 
réglementaires particulières aux départements du Bas-Rhin, du Haut-Rhin et de la Moselle «peuvent demeurer en vigueur; qu'à défaut de leur abrogation ou de leur harmonisation avec le droit commun, ces dispositions particulières ne peuvent être aménagées que dans la mesure où les différences de traitement qui en résultent ne sont pas accrues et que leur champ d'application n'est pas élargi ${ }^{51} »$. Cette règle jurisprudentielle ne protège donc pas le droit local de toute incompatibilité avec le droit constitutionnel, mais seulement des incompatibilités fondées sur le principe d'égalité et à la condition qu'elles n'aient pas fait, après l'entrée en vigueur de la Constitution de 1946, l'objet d'aménagements qui en ont accru la spécificité ${ }^{52}$.

Cette jurisprudence n'est donc que d'un intérêt limité pour une discussion qui s'articule autour du principe de laicité. De plus, la condition de ne pas procéder à des "aménagements nouveaux» est difficile à cerner dans le domaine de l'enseignement de la théologie: si l'on considère que la légalité de celui-ci est fondée sur la non-application de la loi de 1905, la création de nouveaux enseignements ne constitue pas une modification des textes applicables, mais seulement leur mise en ouvre ${ }^{53}$.

\subsubsection{LE DROIT LOCAL PROTÉGÉ PAR L'INTENTION DU CONSTITUANT}

Le Conseil constitutionnel a estimé qu'il « ressort tant des travaux préparatoires du projet de la Constitution du 27 octobre 1946 relatifs à son article $1^{\text {er }}$ que de ceux du projet de la Constitution du 4 octobre 1958 qui a repris la même disposition, qu'en proclamant que la France est une "République [...] laique", la Constitution n'a pas pour autant entendu remettre en cause les dispositions législatives ou réglementaires particulières applicables dans plusieurs parties du territoire de la République lors de l'entrée en vigueur de la Constitution et relatives à l'organisation de certains cultes et, notamment,

51. Cons. const., 5 août 2011, $\mathrm{n}^{\circ}$ 2011-157 QPC, SOMODIA.

52. Le principe fondamental reconnu par les lois de la République dégagé par la jurisprudence constitutionnelle couvre la rupture d'égalité qui pourrait exister du fait de l'existence de la législation locale au regard de la législation générale. Il ne concerne pas des ruptures d'égalité au sein de la législation locale, par exemple le fait que des enseignements théologiques existent pour certains cultes et pas pour d'autres.

53. Faire usage de la licéité d'un enseignement théologique universitaire pour des formes de théologie non prises en considération jusque-là ne modifie pas le contenu de la norme appliquée. Pour écarter toute incompréhension, il faut rappeler également que la législation relative aux cultes dits statutaires (ou reconnus) est distincte et indépendante de celle concernant l'enseignement universitaire. Il n'y a pas de fondement juridique au lien parfois établi entre culte «reconnu » et enseignement théologique à l'Université publique. 
à la rémunération de ministres du culte ${ }^{54} »$. Il résulte de cette analyse que les dispositions caractérisant le régime local des cultes sont restées en vigueur nonobstant leur éventuelle incompatibilité avec les éléments constitutifs du principe constitutionnel de laïcité. Le Conseil constitutionnel a utilisé ce raisonnement pour écarter le grief relatif au fait que la législation locale prévoit de salarier des agents d'institutions cultuelles. Il pourrait l'utiliser également en ce qui concerne l'enseignement de la théologie dans un cadre universitaire public s'il devait retenir l'inconstitutionnalité de celui-ci. De même, pour autant que l'on voudrait déduire du principe constitutionnel de laïcité la non-licéité de la convention de 1902, le principe dégagé par le Conseil constitutionnel pourrait protéger cette particularité locale d'une remise en cause tirée du droit constitutionnel.

Toutefois, comme le raisonnement du Conseil constitutionnel se fonde sur l'intention du constituant en 1946 et 1958, les modifications ultérieures ne sont pas nécessairement couvertes. On peut être tenté d'introduire dans la jurisprudence APPEL du 23 février 2013 la réserve instituée par la décision SOMODIA du 5 août 2011, bien qu'elles portent sur des objets différents (principe d'égalité dans un cas et principe de laïcité dans l'autre) et limiter les deux à la sauvegarde de l'acquis. Mais comme déjà précisé, il faut entendre par modifications du régime local non couvertes par cette jurisprudence les changements de type législatif ou réglementaire et non pas l'application d'une base juridique existante à de nouvelles activités: la création d'un enseignement de théologie musulmane, par exemple, ne constituerait pas une extension du droit local, mais la mise en œuvre de celui-ci avec un nouvel objet.

Quoi qu'il en soit, au plan pratique, les résultats de cette investigation sont minces: puisqu'on a vu que l'enseignement de la théologie à l'université n'est en contradiction avec aucun principe constitutionnel, les mécanismes de sauvegarde susmentionnés sont sans objet pour le droit local de l'enseignement de la théologie et ne pourraient avoir une importance que pour la sauvegarde de la convention de $1902^{55}$.

En conclusion, au regard de la question de la création à l'Université publique d'enseignements nouveaux de théologie, voire de la création de facultés de théologie musulmane, que ce soit en Alsace-Moselle ou dans

54. Cons. const., 21 févr. 2013, n 2012-297 QPC, précit.

55. À l'exclusion de celle de 1974, si on devait analyser celle-ci comme une novation non couverte par les jurisprudences ayant institué une sauvegarde du droit local existant. 
le reste de la France, aucun principe constitutionnel ne s'y oppose. Inversement, sur le plan juridique, en dehors de la non-application de la loi de 1905, dont on a vu la portée limitée en la matière, le droit alsacien-mosellan ne comporte pas pour de telles innovations de bases juridiques originales, qui seraient plus favorables que le droit général. Ce n'est que le contexte intellectuel et culturel alsacien-mosellan qui présente un cadre plus accueillant à une telle initiative en raison de l'existence des facultés de théologie catholique et protestante, ainsi que d'une tradition historique d'intérêt des autorités publiques pour la formation des cadres religieux. 